\title{
prosthetics
}

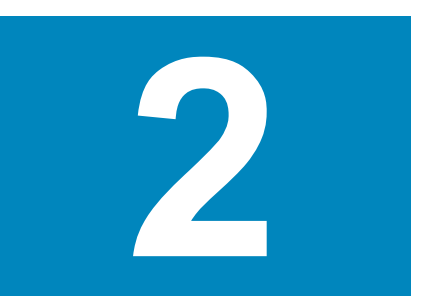

\section{The removable partial denture equation}

\author{
J. C. Davenport, ' R. M. Basker, ${ }^{2}$ J. R. Heath, ${ }^{3}$ J. P. Ralph, ${ }^{4}$ and P-O. Glantz, ${ }^{5}$
}

\author{
This article describes the benefits and risks of \\ providing RPDs. It emphasises the importance of \\ co-operation between the dental team and patient \\ to ensure that the balance of this 'equation' is in \\ the patient's favour.
}

\author{
In this part, we will discuss \\ - The benefits of RPDs \\ - Tissue damage associated with RPDs \\ - Preserving oral health \\ - The importance of teamwork
}

$\mathrm{T}$ The title of this part of the series requires immediate explanation. The term 'equation' refers to the balance that must be struck between the good and the bad which can arise from the wearing of RPDs. In this chapter we explore the benefits which can be conferred on patients by RPDs and, at the same time, highlight the possible risks of tissue damage that can be associated with such prostheses.

Every prosthetic treatment is associated with the placement of a foreign object (the prosthesis) in the mouth of the patient. As a direct consequence of such placement the burden on the tissues in the oral cavity will be increased. For example, plaque more readily accumulates on alloplastic materials than biologic ones. Furthermore, even non-toxic materials will release small amounts of their components into the oral cavity. To justify prosthetic treatment and to ensure that it is beneficial to the patient, the need for such treatment must be established, the patient must be appropriately motivated, and the dentures properly designed, constructed and maintained. Thus the initial step in determining if prosthetic treatment is indicated must always be the assessment of:

- The patient's wishes and concerns

- The relevant dental and medical history

- The results of the extra-oral and intra-oral examinations

- Oral hygiene habits and status
- The essential oral functions of appearance, mastication and speech.

It is only after this analysis has been completed that the decision of whether or not to treat a particular patient can be taken. For example, prosthetic treatment must not begin until it has been verified that there is a significant reduction in one or more of the essential oral functions. A simple determination of the number and position of the remaining teeth is not a sufficient foundation for making the decision of whether or not to initiate treatment. If it is indicated, a treatment plan is then devised identifying the various stages and the most appropriate type of prosthesis.

\section{Benefits of RPDs}

The potential benefits of RPDs which will be considered in this section are their contribution to the following.

- Appearance

- Speech

- Mastication

- Maintaining the health of the masticatory system:

— preventing undesirable tooth movement

— improving distribution of occlusal load

- Preparation for complete dentures.

\footnotetext{
$1^{*}$ Emeritus Professor, University of Birmingham, UK; ${ }^{2}$ Professor of Dental Prosthetics, University of Leeds and Consultant in Restorative Dentistry, Leeds Teaching Hospitals NHS Trust, Leeds, UK; ${ }^{3}$ Honorary Research Fellow, University of Manchester (Formerly Senior Lecturer in Restorative Dentistry, University of Manchester) and Consultant in Restorative Dentistry, Central Manchester Healthcare Trust, Manchester, UK; ${ }^{4}$ Consultant in Restorative Dentistry, Leeds Teaching Hospitals NHS Trust and Senior Clinical Lecturer, University of Leeds and Honorary Visiting Professor, Centre for Dental Services Studies, University of York, York, UK; ${ }^{5}$ Professor of Prosthetic Dentistry, Consultant in Prosthetic Dentistry, Faculty of Odontology, University of Malmo, Sweden

${ }^{*}$ Correspondence to: 5 Victoria Road, Harborne, Birmingham B17 OAG email:john.davenport@btclick.com

REFEREED PAPER

(C) British Dental Journal 2000; 189: 414-424
}

\section{New publications:}

All the parts which comprise this series (which will be published in the BDJ) have been included (together with a number of unpublished parts) in the book A Cinical Guide to Removable Partial Dentures (ISBN 0-904588-599) and A Clinical Guide to Removable Partial Denture Design (ISBN 0-904588-637). Available from Macmillan on 01256302699

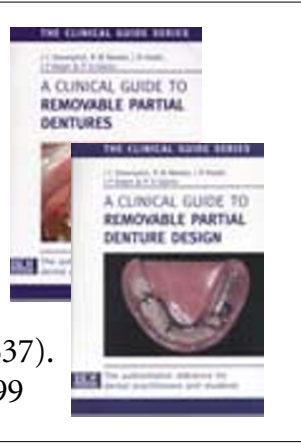




\section{PRACTICE prosthetics}

\section{Fig. I-Appearance}

The restoration of the missing UR2 (I2), URI (II) and ULI (2I) undeniably benefit this patient, an 18 year-old girl, and the motivation to wear the denture is understandably strong. Particular attention has been paid to the appearance of the denture by the careful choice of artificial teeth and design of the flange. A natural appearance has been created by using a 'veined' acrylic, by reproducing the pre-extraction form of alveolar ridge and by making the distal margin of the flange thin and irregular, thus masking the transition from flange to adjacent mucosa.

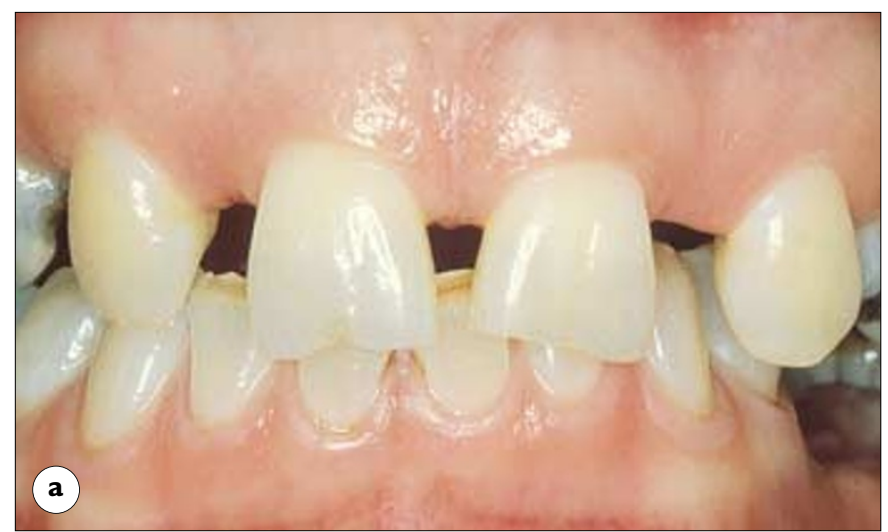

Fig. $2 a$ and $b-$ Appearance

Not only may an RPD help to restore appearance but it may actually improve it.

(a) This patient's maxillary lateral incisors had never developed and she was concerned about the spacing of the anterior teeth.

(b) The combination of orthodontic movement of the central incisors and the provision of RPDs improved the appearance.

\section{Fig. 3 - Appearance}

If an incisor is not replaced soon after extraction, successful treatment at a later date may be compromised. Here, the adjacent teeth have drifted into the unrestored ULI (2I) space. The reduced space does not allow for an artificial tooth of a realistic size to be used on a denture. If a reasonable aesthetic result is to be obtained the space must be reestablished by orthodontic treatment.

\section{Fig. 4 - Speech}

The loss of maxillary anterior teeth may prevent the clear reproduction of certain sounds, particularly the ' $F$ ' and ' $\mathrm{V}$ ' which are made by the lower lip contacting the edges of the maxillary incisors. The replacement of missing maxillary anterior teeth will make a significant contribution to the quality of speech.
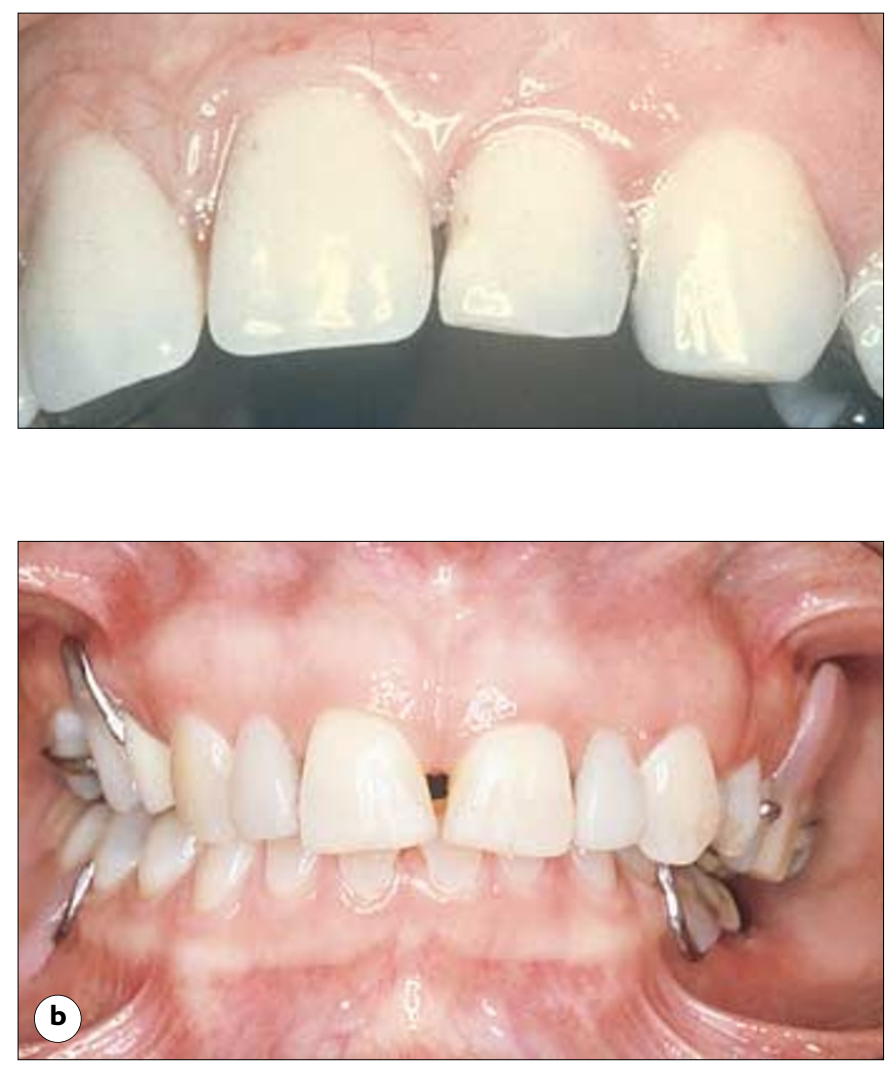

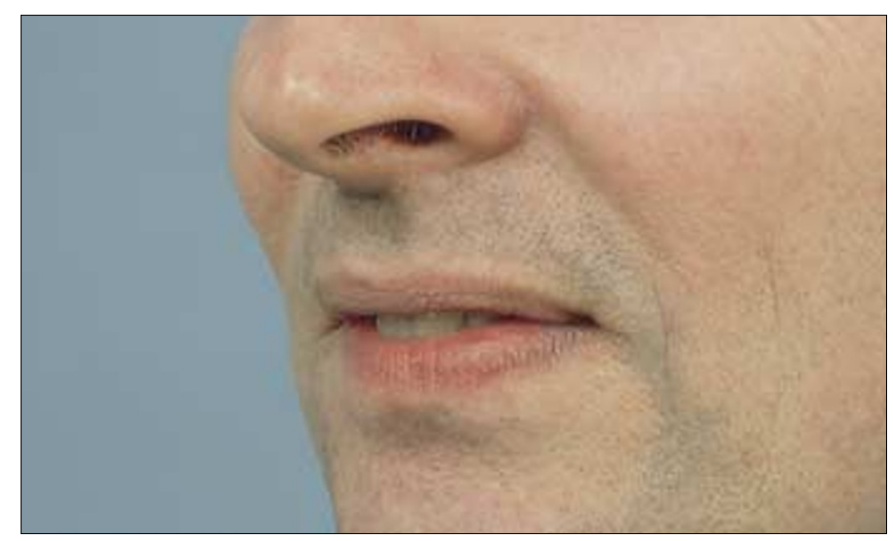




\section{prosthetics}
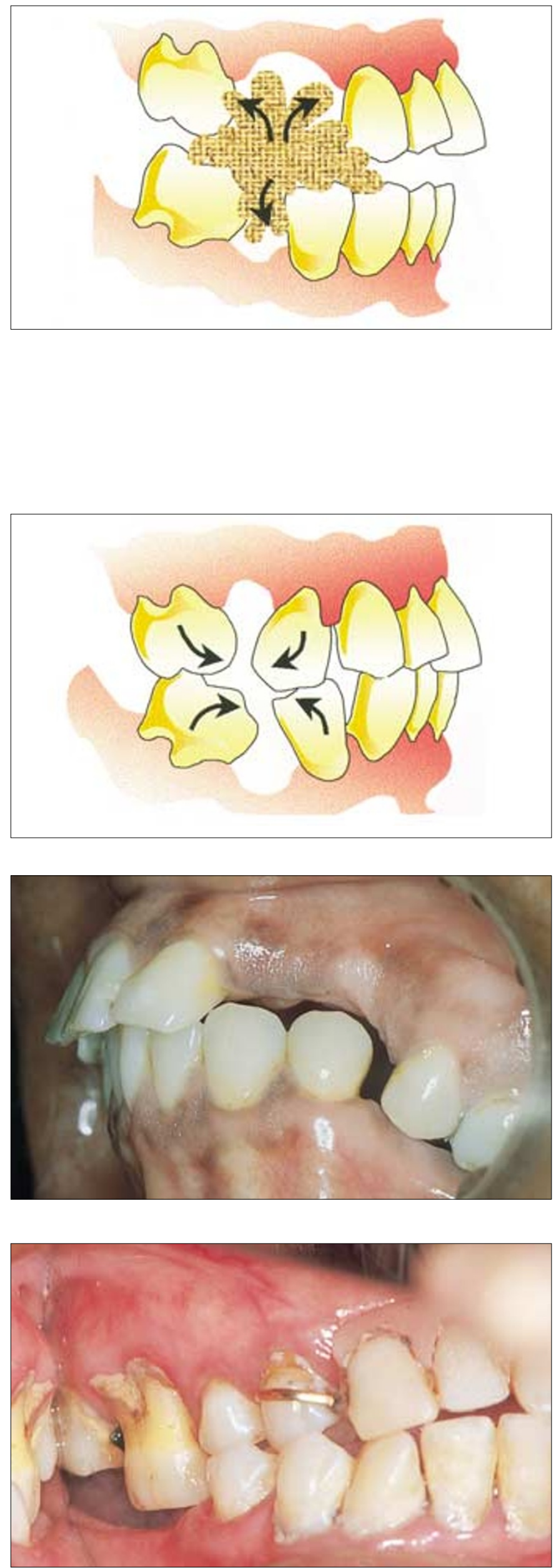

\section{Fig. 5 - Mastication}

With modern foods and methods of preparation it is unlikely that a patient will suffer from malnutrition even though a large number of teeth are missing. However, the gaps that arise through the loss of posterior teeth reduce the efficiency of mastication: the bolus of food is allowed to slip into the edentulous areas and thus escape the crushing and shearing action of the remaining teeth. An RPD will prevent this escape of the bolus and thus contribute to efficient mastication.

Maintaining the health of the masticatory system

The provision of an RPD can make a positive contribution to oral health by preventing, or minimising, the undesirable consequences of tooth loss, as described in the following paragraphs.

\section{Fig. 6 - Preventing undesirable tooth movement}

When teeth are lost from a dental arch the teeth adjacent to the edentulous space may tilt and move into that space. This drifting of teeth opens up further spaces which increase the opportunity for food impaction and plaque formation, encouraging inflammation of the periodontal tissues and decalcification of the proximal surfaces of the teeth. Inevitably, the longer such spaces remain unrestored, the greater the chance of tooth movement. When teeth are lost from an opposing arch over-eruption may occur with similar deleterious effects on the oral health. However, if tooth movement has not occurred in spite of the teeth being lost some years previously, it can be assumed that it is not going to occur subsequently.

\section{Fig. 7 - Preventing undesirable tooth movement}

The long-term absence of antagonists has resulted in over-eruption of maxillary and mandibular teeth. The teeth are virtually contacting the opposing edentulous ridges creating major problems if RPDs have to be provided.

\section{Fig. 8 - Preventing undesirable tooth movement}

In this example, UR6 (I6) has over-erupted to such an extent that it has lost most of its bony support. Extraction of the tooth is inevitable. 


\section{PRACTICE prosthetics}

\section{Fig. 9 - Improved distribution of occlusal load} on the remaining teeth. In this example there is existing periodontal periodontal attachments of the maxillary anterior teeth, which have become increasingly mobile and have drifted labially.
The loss of a large number of teeth puts an increasing functional burden disease. The increased functional load has hastened the destruction of the
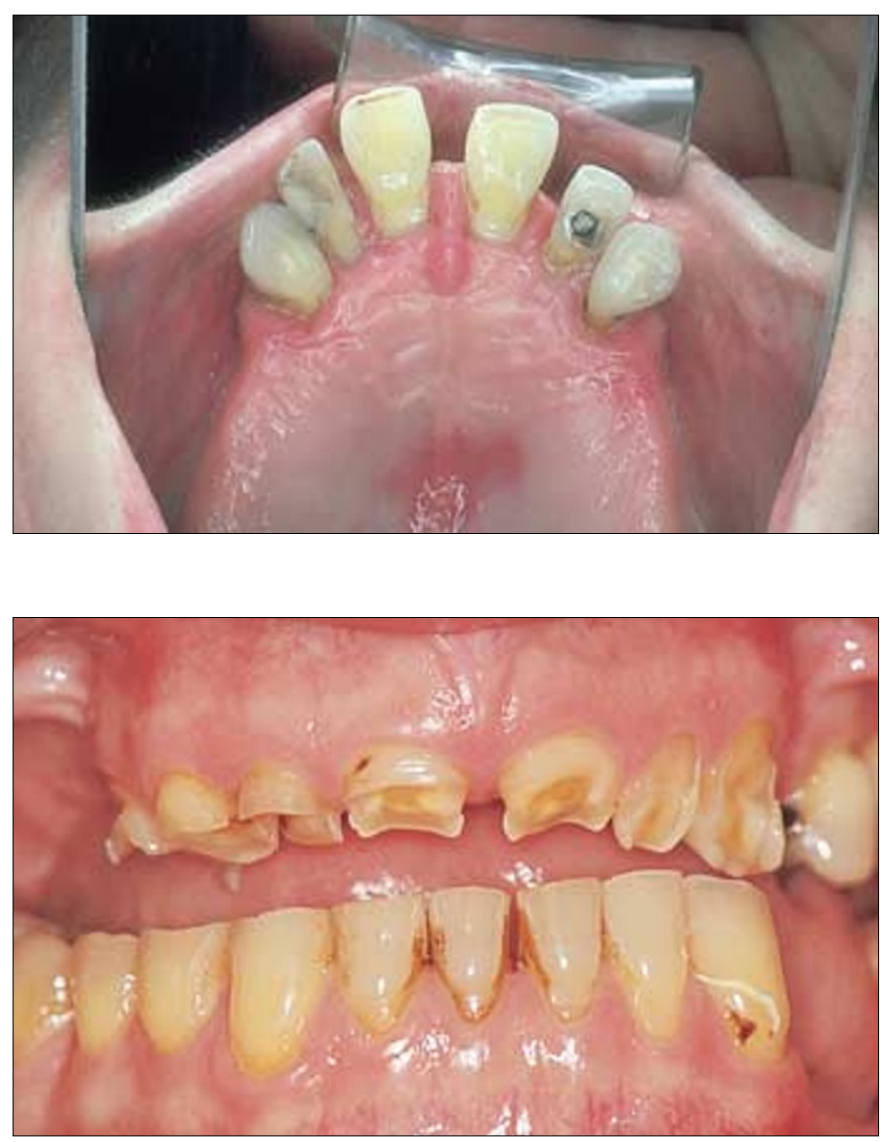

Fig. 10 - Improved distribution of occlusal load

If the periodontal attachments of the remaining teeth are healthy, the increased load may result in excessive tooth wear or may cause damage to existing restorations. The restoration of gross loss of tooth substance, as in this example, is likely to involve complex and prolonged treatment.
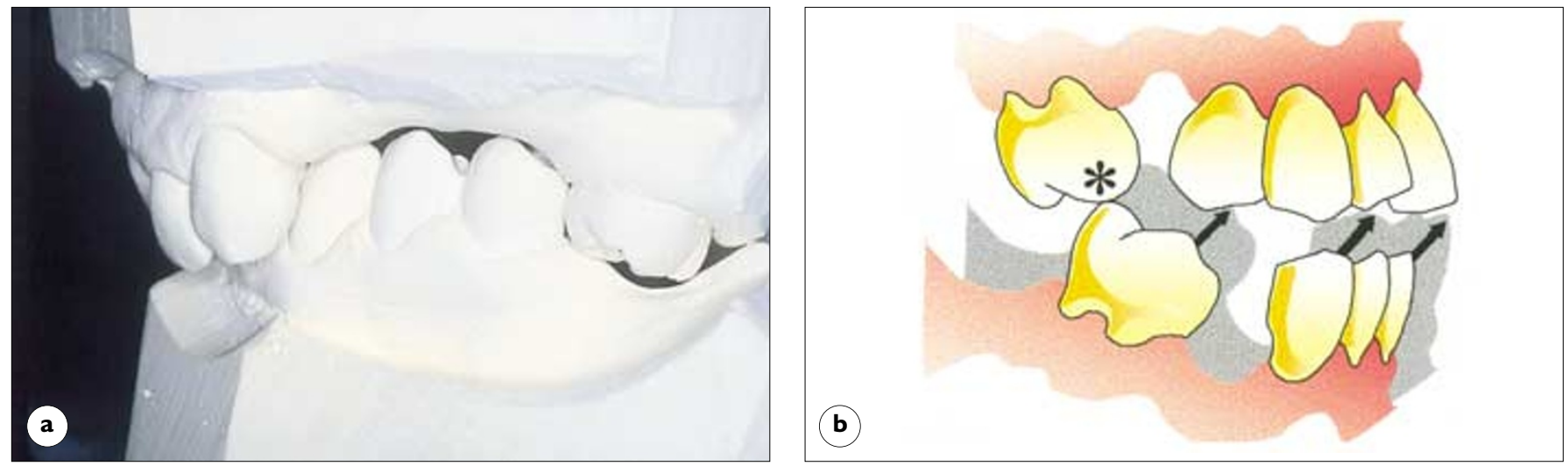

Fig. I I $a$ and $b$ - Improved distribution of occlusal load

Over-eruption of a tooth may place it in such a position that it bears the brunt of the load on initial contact or in excursive movements of the mandible and therefore it may well be subjected to excessive force. In addition, where over-eruption of a tooth has created an occlusal interference (*), the patient may modify the habitual movement patterns of the mandible in order to avoid the interfering contact. Although such a modification may reduce the load applied to the tooth, the changed pattern in activity of the mandibular musculature may subsequently produce muscular dysfunction.

From the foregoing examples it will be appreciated that if tooth loss is restored in sufficient time to prevent tooth movement, or to avoid excessive stress being placed on the remaining structures, the subsequent health of the oral tissues can benefit considerably.

However, the point should be made that severe damage to the existing structures is not an inevitable consequence of tooth loss. The implications of this statement will become apparent later in this section when the damaging effects of the dentures themselves are described.

\section{Preparation for complete dentures}

Most of this book is devoted to the treatment of patients who are expected to retain their remaining natural teeth for a considerable number of years, thus allowing the RPD to be regarded as a long-term restoration. But we should remember those patients whose remaining teeth carry a relatively poor prognosis and for whom, in due course, complete dentures are inevitable. If simple acrylic RPDs are provided, the patient is able to serve a prosthetic 'apprenticeship' with appliances which receive some stability from the few remaining teeth. In the fullness of time these transitional dentures become more extensive as further teeth are extracted and the patient is gradually eased into the totally artificial dentition. This form of transitional treatment can be of considerable benefit, especially for the elderly patient. 


\section{prosthetics}
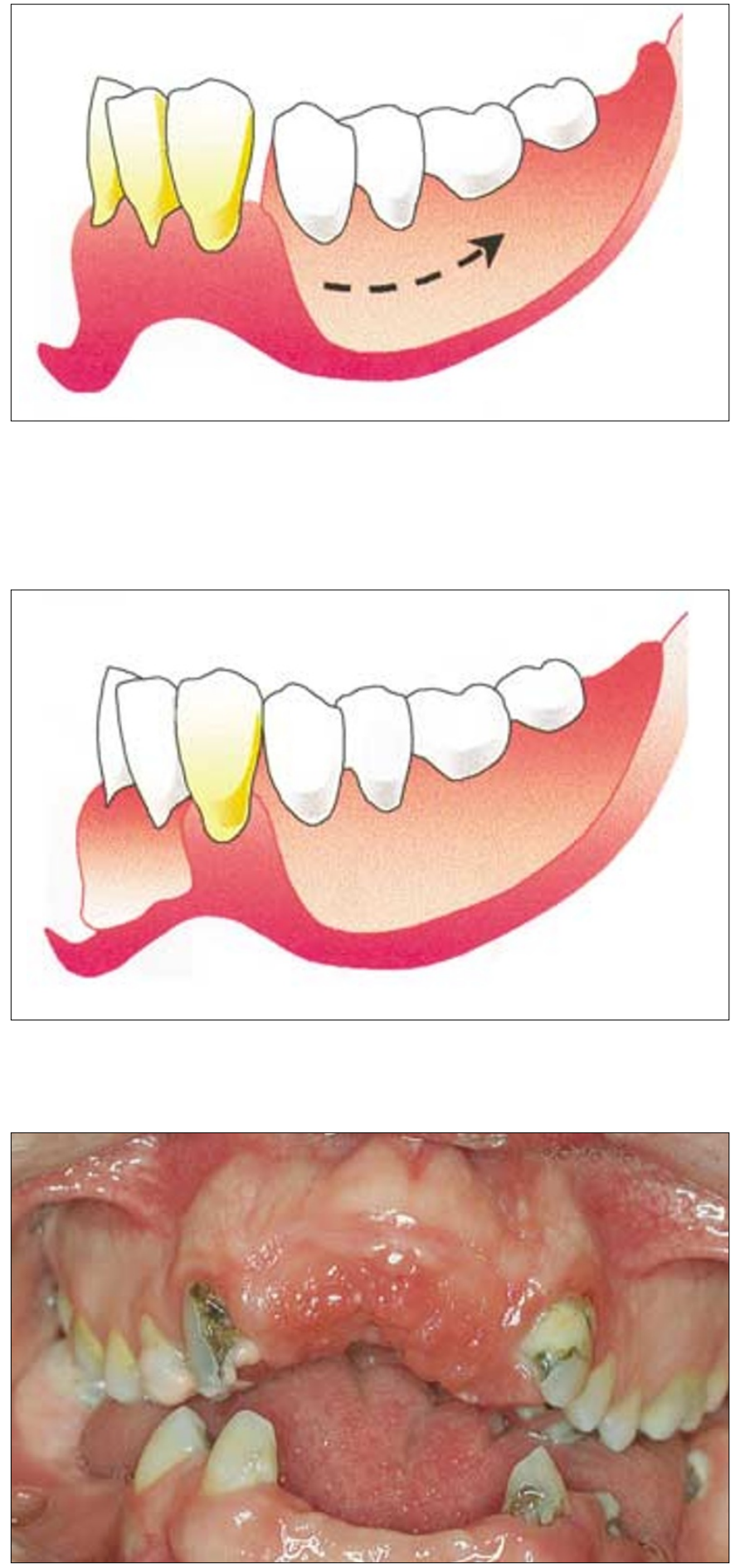

\section{Causes of damage related to the wearing of RPDs}

Harmful effects can arise from the wearing of RPDs in a variety of ways: from the plaque which is likely to accumulate around any RPD; from direct trauma by individual components of the denture; from excessive functional forces which will be transmitted by an ill-designed prosthesis and from errors in the occlusion.

If the patient, with the help of the dental team, can maintain optimal plaque control the hygiene-related complications of

\section{Fig. 12 - Preparation for complete dentures}

The location of the remaining teeth plays an important part in the success of such a transitional denture. It is common for the six mandibular anterior teeth to be the last remaining teeth in the mandible. A denture restoring the posterior teeth is frequently not worn by the patient for the following reasons. Firstly, the denture may be unstable because there is little resistance to its displacement in a posterior direction; secondly, there is very little motivation to wear the denture as the anterior teeth are still present.

\section{Fig. 13 - Preparation for complete dentures}

If, instead of extracting all the teeth, the canines are retained, the denture will be more stable. When the denture replaces anterior teeth it is very much more likely to be worn and thus the patient is likely to gain greater benefit from the transitional denture.

\section{Fig. 14 - Preparation for complete dentures}

It should be remembered that the transitional RPD is being placed in a mouth where existing dental disease is only poorly controlled or is uncontrolled. As will be seen in the next section, the very presence of a denture aggravates the situation. If the mouth is not inspected regularly to identify treatment needs as they arise, there is the likelihood of acceleration of tissue damage, which may prejudice the eventual complete denture foundation.

In this case the inflammation and hyperplasia of the palatal mucosa was so severe that surgery had to be performed before further prosthetic treatment could be undertaken.

wearing RPDs, such as caries and periodontal disease, can be avoided. However, frequent technical maintenance of RPDs is still required if optimal oral function and health are to be preserved.

When tissue damage does occur it is sometimes referred to as the 'biological price' of wearing RPDs. The possible causes of damage and their sequelae are summarised in the following table and are discussed in more detail in the subsequent sections. 


\begin{tabular}{|c|c|c|c|c|}
\hline Table I & nary of damage & $t$ may result from weari & RPD & \\
\hline Causes & Teeth & Periodontal tissues & Edentulous areas & Muscles of mastication \\
\hline Plaque accumulation & $\begin{array}{l}\text { Decalcification } \\
\text { and caries }\end{array}$ & $\begin{array}{l}\text { Inflammation of gingival } \\
\text { tissues } \\
\text { Progression to underlying } \\
\text { structures }\end{array}$ & $\begin{array}{l}\text { Inflammation of mucous } \\
\text { membrane }\end{array}$ & \\
\hline $\begin{array}{l}\text { Direct trauma from } \\
\text { components }\end{array}$ & $\begin{array}{l}\text { Abrasion and } \\
\text { fracture of } \\
\text { teeth or } \\
\text { restorations }\end{array}$ & $\begin{array}{l}\text { Inflammation of gingival } \\
\text { tissues } \\
\text { Progression to underlying } \\
\text { structures }\end{array}$ & $\begin{array}{l}\text { Localised inflammation } \\
\text { of mucous membrane. } \\
\text { Denture-induced } \\
\text { hyperplasia }\end{array}$ & \\
\hline $\begin{array}{l}\text { Transmission of } \\
\text { excessive functional } \\
\text { forces }\end{array}$ & & $\begin{array}{l}\text { (a) Tooth mobility } \\
\text { (b) Aggravation of existing } \\
\text { periodontal disease }\end{array}$ & $\begin{array}{l}\text { Inflammation of mucous } \\
\text { membrane } \\
\text { Resorption of bone }\end{array}$ & \\
\hline Occlusal error & & $\begin{array}{l}\text { (a) Tooth mobility } \\
\text { (b) Aggravation of existing } \\
\text { periodontal disease }\end{array}$ & $\begin{array}{l}\text { Inflammation of mucous } \\
\text { membrane } \\
\text { Resorption of bone }\end{array}$ & Muscle dysfunction \\
\hline
\end{tabular}

\section{Increased plaque accumulation}

A considerable amount of research effort has been directed towards an understanding of the relationship between plaque accumulation and the wearing of RPDs. It is possible that the presence of a denture influences the quality of the plaque; it certainly affects the quantity. Not only does more plaque accumulate around the teeth in the jaw in which the denture is placed, but also more is found around the teeth in the opposing jaw unless the patient is instructed in meticulous oral hygiene procedures.
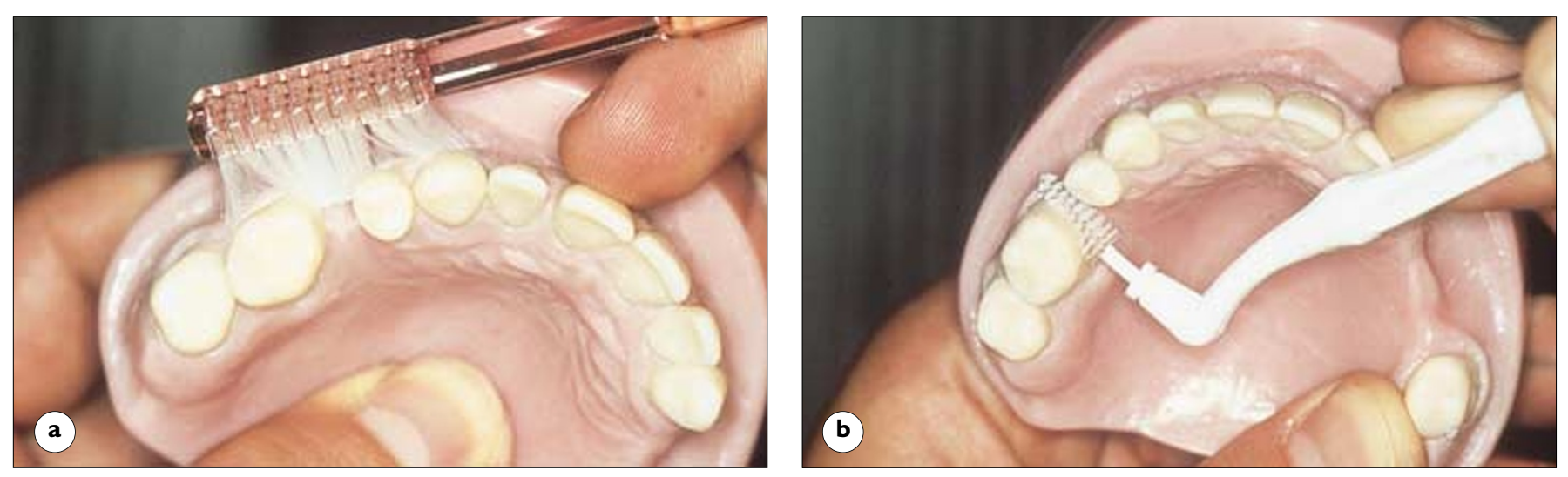

Fig. I $5 a$ and $b$ - Increased plaque accumulation

The areas that tend to collect most plaque are the proximal surfaces of abutment teeth adjacent to the saddle.

(a) These surfaces are difficult to clean when using a conventional toothbrush.

(b) An interdental brush cleans the proximal surfaces more effectively. 


\section{PRACTICE prosthetics}
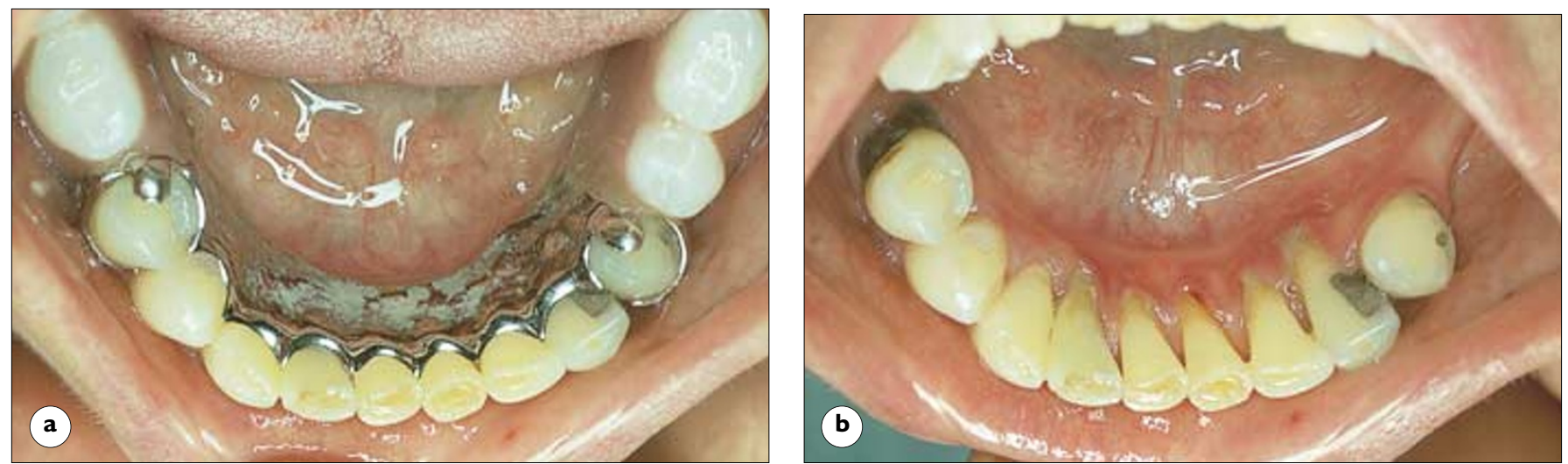

Fig. I6a and $b$ - Increased plaque accumulation

The design of the denture may have a significant effect on plaque accumulation. For example, it has been shown that more plaque collects under a lingual plate than under a lingual bar.

(a) The lingual plate is well supported on the natural teeth and fits well against tooth surfaces.

(b) However, gingival inflammation has been caused by the increased accumulation of plaque.

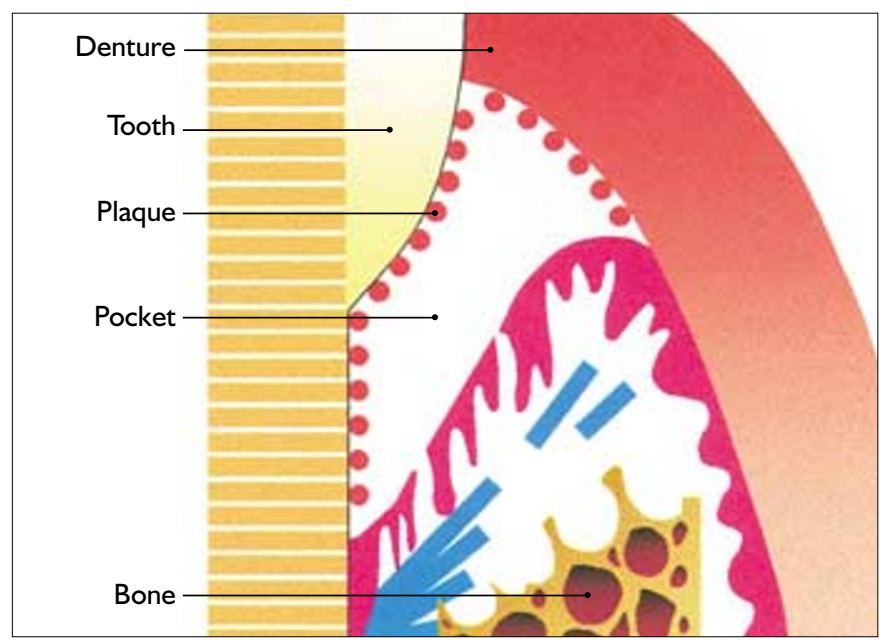

Fig. I 7 - Increased plaque accumulation

If the plaque is allowed to persist, the inflammatory process will progress to the deeper tissues, resulting in a chronic periodontitis. The periodontal attachment is progressively destroyed, a periodontal pocket develops and the investing alveolar bone is lost.

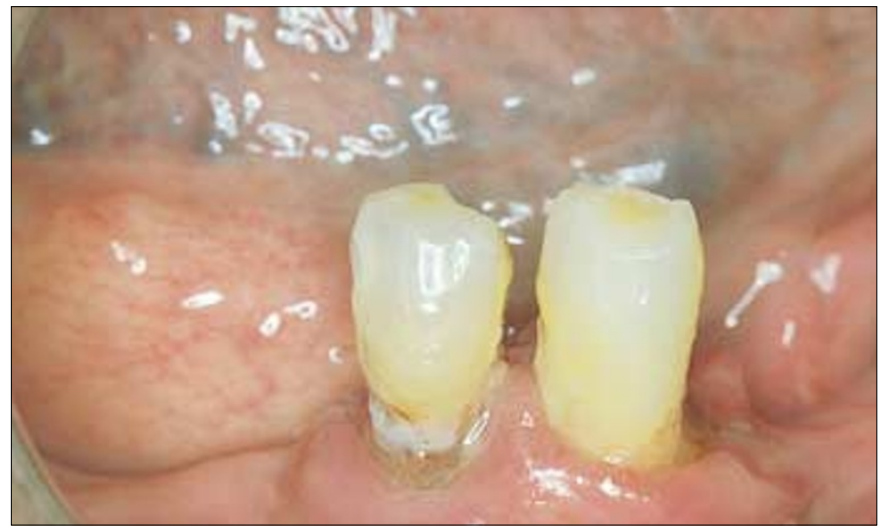

Fig. 18 - Increased plaque accumulation

Unless the increased accumulation of plaque is prevented, root caries is likely. This is a problem that will increase as more patients continue to wear RPDs into old age. Root caries is strongly associated with gingival recession and the use of gingivally-approaching clasps in patients who are at risk because of a cariogenic diet and poor plaque control. 
Fig. 19 - Increased plaque accumulation

If plaque is allowed to persist on the denture impression surface, a generalised inflammation, called denture stomatitis, may occur. Typically the extent of the inflammation is demarcated by the outline of the palatal connector. This condition is discussed more fully in Part II.
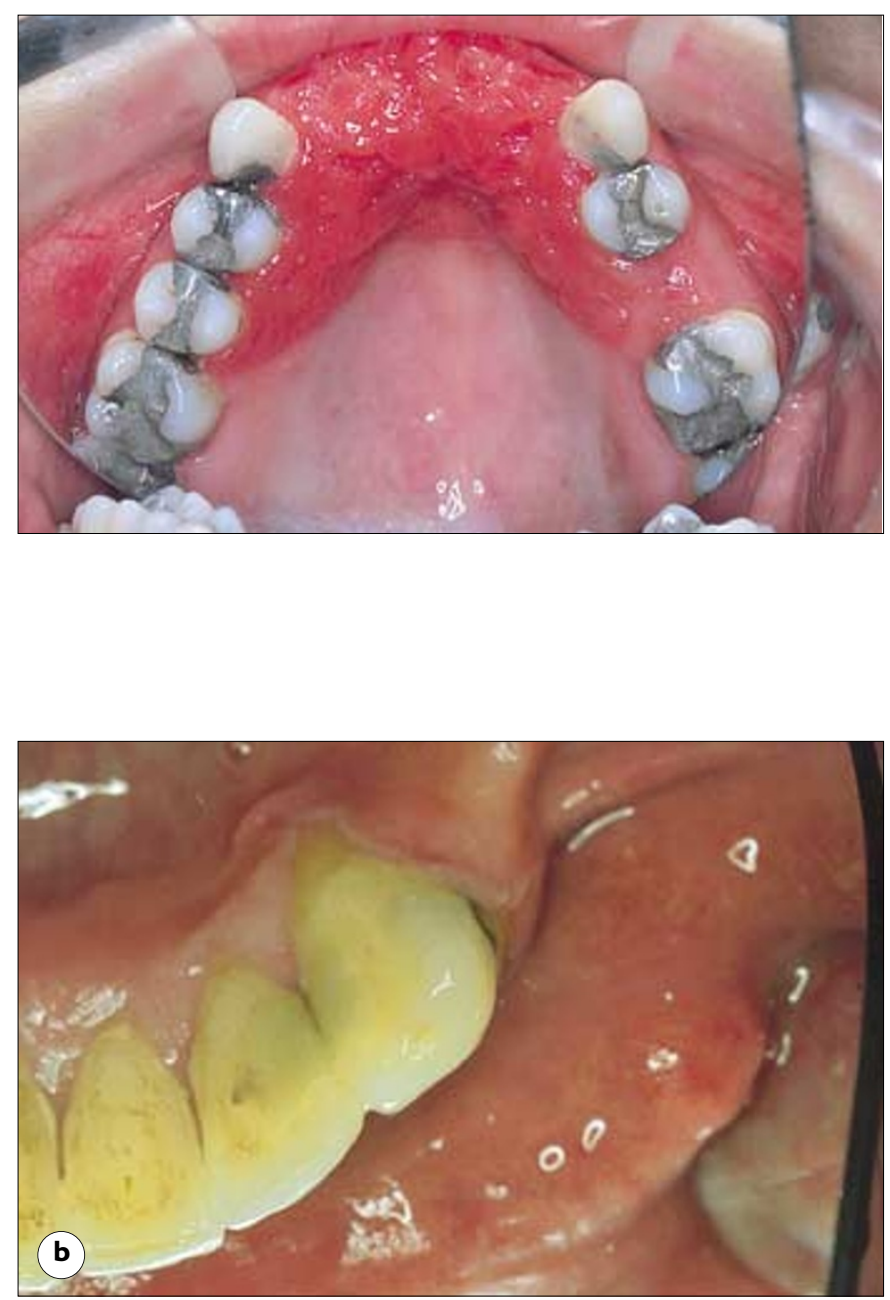

Fig. 20a and b - Direct trauma from components

The oral mucosa is vulnerable to direct trauma from components of dentures.

(a) In this instance the lingual bar has been positioned too close to the gingival margin. The continuous clasp offers only limited tooth support for the denture.

(b) The denture has sunk into the tissues, stripping away the gingival tissues on the distal and lingual aspects of LL3 (33).

There is no evidence for the contention that a clasp arm may wear away the enamel surface to a degree that is significant clinically. However, the movement of a clasp arm may wear the surface of restorative materials.

Transmission of excessive force

Functional forces are transmitted by an RPD to the tissues with which it is in contact. If a denture is supported primarily by the natural teeth most of the forces will be transmitted to the alveolar bone through the fibres of the periodontal ligament. Bearing in mind the orientation of most of these fibres, it will be appreciated that the forces are tensile in nature and are dissipated over a relatively large area. A very different state of affairs exists when a denture is supported only by the mucosa. Here the forces, largely compressive in nature, are transmitted over a more restricted area. 


\section{PRACTICE}

\section{prosthetics}
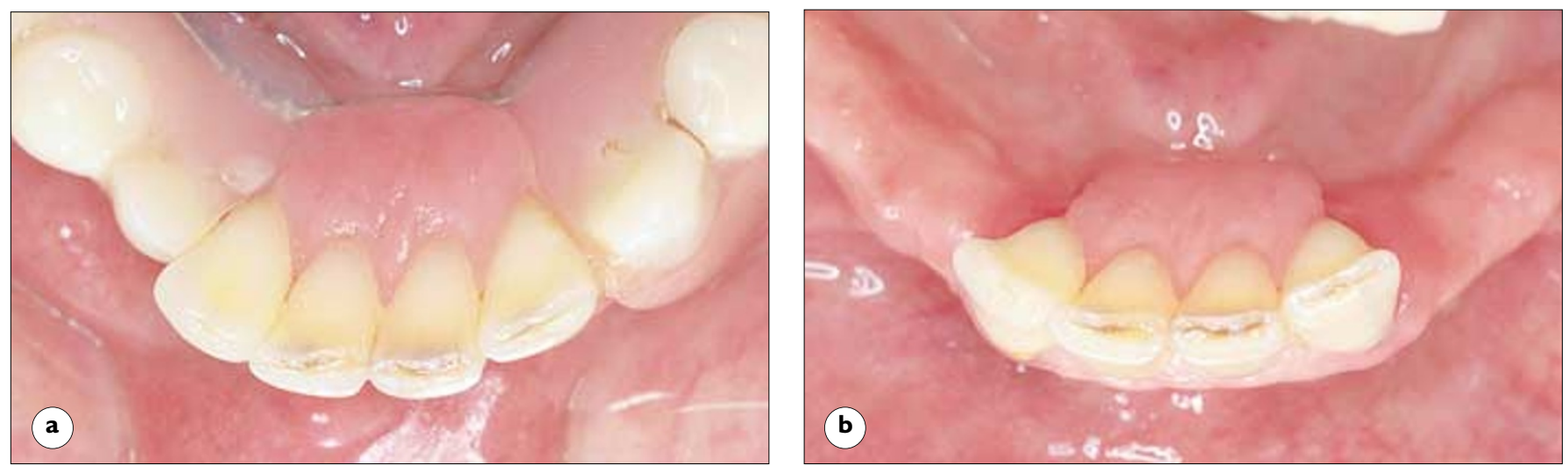

Fig. 2l $a$ and $b$ - Transmission of excessive force

If the forces transmitted to the mucosa and bone of the edentulous area are excessive, the mucosa will become inflamed and the bone will resorb. The obvious consequence of bone resorption is an irreversible loss of part of the denture foundation.

(a) In this example the denture is supported only on the tissues of the edentulous area. It has caused resorption of the bone to such an extent that the lingual bar connector has been pushed down towards the floor of the mouth.

(b) The amount of bone that has been destroyed is apparent when the denture is removed.

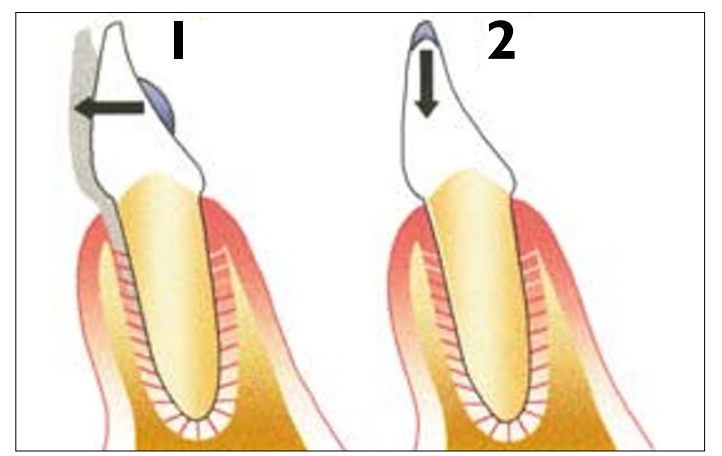

Fig. 22 - Transmission of excessive force

If the design of the denture is such that it transmits excessive force to a tooth there is every chance that the tooth will become mobile.

In this example the incorrectly designed cingulum rest (I) transmits a horizontal force to the canine tooth. Such horizontal forces are especially damaging to the periodontal tissues. The incisal rest (2) transmits a more favourable vertical load.

Where periodontal changes are restricted to the marginal gingivae, elimination of excessive force will usually allow the periodontal attachment to return to a normal healthy state. Where the supporting structures have been affected by periodontal disease there is unlikely to be complete resolution.

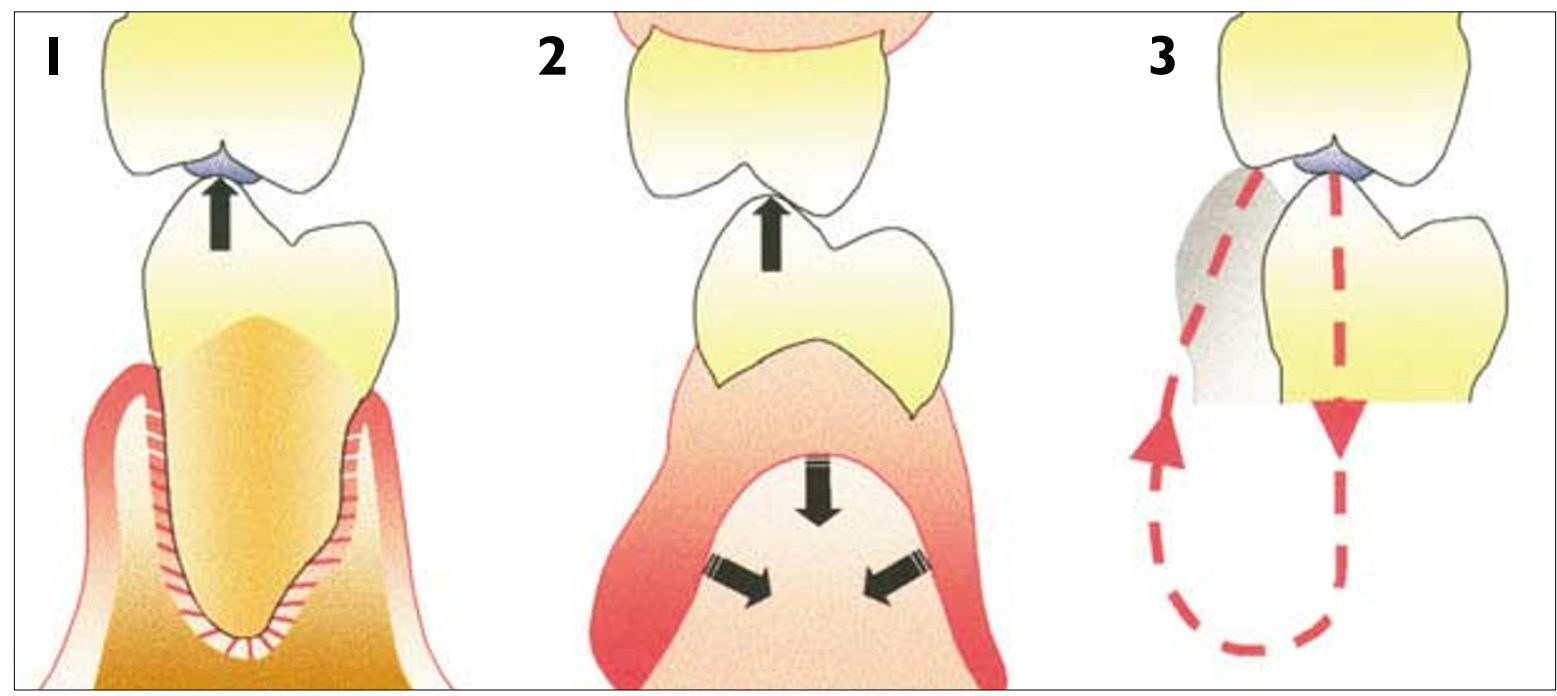

\section{Fig. 23 - Occlusal error}

If the occlusal surface of the RPD is not designed correctly, normal jaw closure may be prevented by a premature occlusal contact. There are three possible sequelae:

(I) If the premature contact is on a natural tooth, damage to the tooth or its periodontal ligament may occur.

(2) If the saddle bears the brunt of the force of closure, there will be localised mucosal inflammation and resorption of the underlying bone.

(3) If the patient attempts to steer the mandible around the premature contact until a more comfortable occlusal position is found, this abnormal closing pattern throws increased demands on certain muscles of mastication, which may result in the patient complaining of facial pain. 


\section{PRACTICE prosthetics}

\section{Balancing the RPD equation}

A number of longitudinal clinical studies of RPDs have shown that the types of damage itemised in the last section may be commonly found amongst wearers of RPDs. Of considerable concern are reports that many patients expressed satisfaction with their dentures, in spite of the fact that dental health had deteriorated markedly. Perhaps this finding is not altogether surprising when we remember the insidious nature of the progression of caries and periodontal disease.

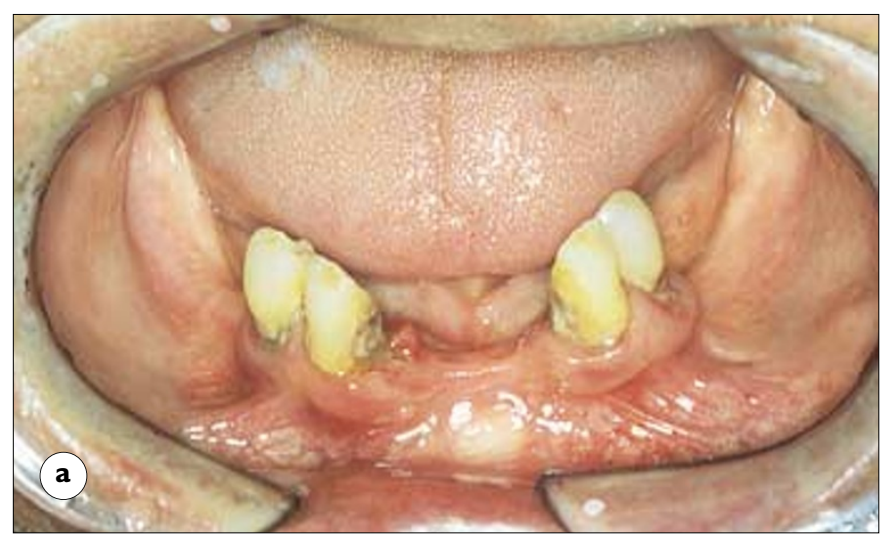

With greater understanding of the relationship between plaque and dental disease and of the importance of plaque control, reports have appeared whose findings make for more encouraging reading. There is now firm evidence that the wearing of RPDs can be compatible with continued oral health. This satisfactory outcome depends upon a three-fold effort, that of the clinician, the dental technician and the patient.

\section{Fig. 24a and $b$-Contribution of the clinician}

The primary responsibility of the dentist and the clinical team is to ensure that the remaining teeth and supporting tissues are restored to a healthy state and that the patient is effectively motivated and instructed in how to maintain this state.

(a) This mouth is not in a fit state to receive an RPD. There is chronic periodontal disease and accumulation of plaque.

(b) This patient has responded well to instruction in oral hygiene and the periodontal tissues are healthy. The dangers of wearing the RPDs are thus minimised.

The second area of responsibility of the clinician is in relation to the design and construction of the denture. Accuracy of the clinical procedures must, of course, be ensured. In addition, the clinician should produce a design based on criteria that have been shown to promote continued oral health:

- Effective support

\section{Fig. 25 - Contribution of the dental technician}

The dental technician's contribution is directed towards the careful translation of the prescribed denture design into the denture itself, and accurate construction and positioning of the denture components. In this instance the inaccurate fit will encourage plaque formation with consequent periodontal disease and caries, thus introducing an unnecessary and avoidable risk to oral health.
- Clearance of gingival margins

- Simplicity

- Rigid connector.

These criteria are considered in greater detail in our BDJ book 'A Clinical Guide to Removable Partial Denture Design'.

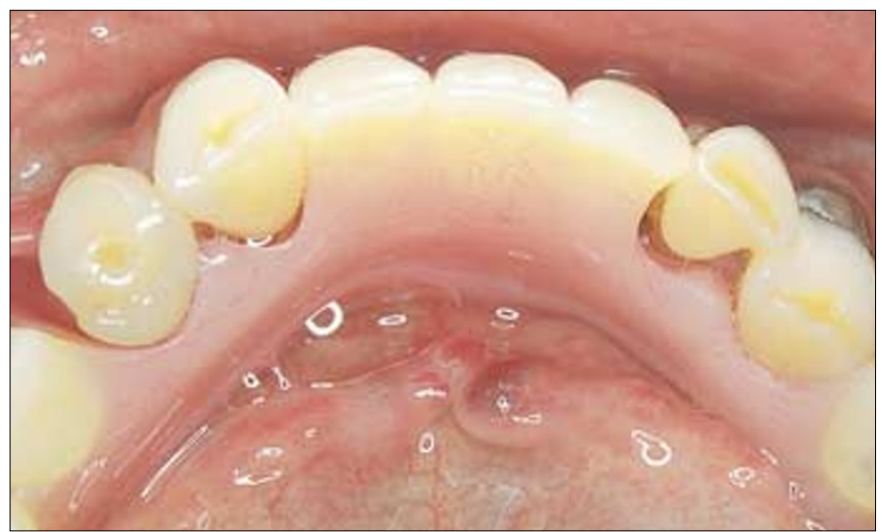




\section{PRACTICE prosthetics}

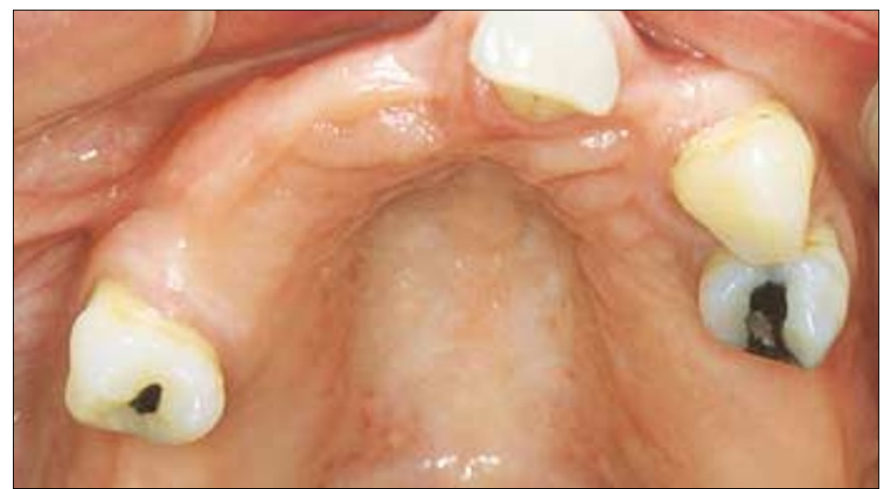

For every patient, when a denture is contemplated, it is the dentist's responsibility to assess the advantages and disadvantages for that particular individual. The level of disadvantage is influenced primarily by the patient's dental awareness and plaque control. When the balance of the equation leans towards

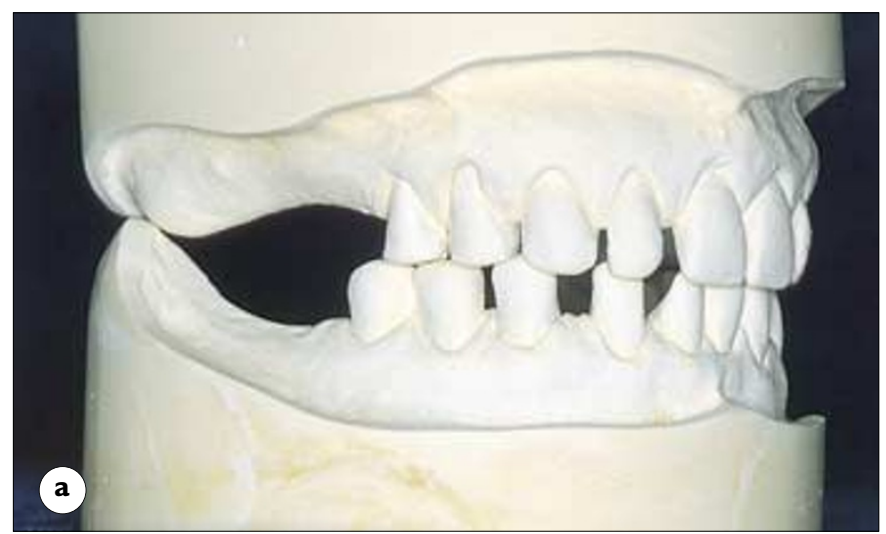

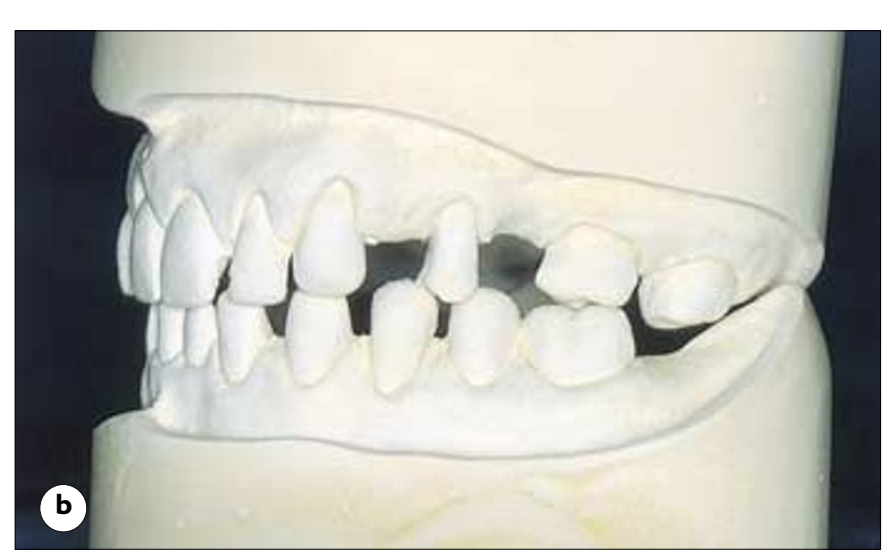

\section{Fig. 26 - Contribution of the patient}

It is probably true to say that a patient who maintains immaculate plaque control and has a good tissue resistance, or 'host response', can be provided with a less than satisfactorily designed denture and still maintain good oral health. Such is the importance of patient factors in the RPD equation. This patient has worn a maxillary RPD for many years. The gingival tissues are healthy and the teeth are well supported by bone; all this in spite of the fact that there is little opportunity to provide tooth support.

disadvantage it is likely that it will be in the patient's best interest that a denture is not prescribed. Of course, where a denture is required to replace an anterior tooth or teeth, the demand from the patient will usually be overwhelming even if the level of plaque control is less than satisfactory.

\section{Fig. 27a and $b$ - Contribution of the patient}

In this mouth the reasons for providing dentures are not overwhelming. There are sufficient teeth at the front of the mouth to satisfy the demands of appearance and speech. There are certainly enough teeth to allow a varied diet to be eaten. Most of the teeth have antagonists in the opposing arch.

If the mouth is well cared for and the patient requests dentures, the RPD equation is favourably balanced.

However, if plaque control is suspect, there is a strong argument for advising against dentures, at least for a few months until the long-term response to oral hygiene advice is ascertained.

\section{Further reading}

RPDs and Oral Health

Bates J F. Plaque accumulation and partial denture design. In Bates J F, Neill D J, Preiskel H W (ed). Restoration of the Partially Dentate Mouth, 225-236. Chicago: Quintessence, 1984.

Berg B. Periodontal problems associated with use of distal extension removable partial dentures - a matter of construction? J Oral Rehabil 1985; 12: 369-379.

Blinkhorn A S. Dental health education: what lessons have we ignored? Br Dent J 1998; 184: 58-59.

Budtz-Jorgenson E. Oral mucosal lesions associated with the wearing of removable dentures. J Oral Path 1981; 10: 65-80.

Carlsson G E, Hedegård B, Koivumaa K K. Studies in partial denture prosthesis IV Final results of a 4-year longitudinal investigation of dentogingivally supported partial dentures. Acta Odont Scand 1965; 23: 443-472.

Chandler J A and Brudvik J S. Clinical evaluation of patients eight to nine years after placement of removable partial dentures. J Prosthet Dent 1984; 51: 736-743.

Germundsson B, Hellman M, Odman P. Effects of rehabilitation with conventional removable partial dentures. Swed Dent J 1984; 8: 171-182.

Gray R J M, Davies S J, Quayle A A. Temporomandibular disorders. a clinical approach. London: British Dental Association, 1995.

MacEntee M I. Biologic sequelae of tooth replacement with removable partial dentures: a case for caution. J Prosthet Dent 1993; 70: 132-134.

McHenry K R, Johansson O B, Christersson L A. The effect of removable partial denture framework design on gingival inflammation - a clinical model. J Prosthet Dent 1992; 68: 799-803.

Orr S, Linden G J, Newman H N. The effect of partial denture connectors on gingival health. J Clin Periodontol 1992; 19: 589-594. 